\title{
Equivalence between two finite element methods for the eddy current problem *
}

\author{
Alfredo Bermúdez ${ }^{\mathrm{a}, 1}$, Bibiana López-Rodríguez ${ }^{\mathrm{b}, 2,3}$, Rodolfo Rodríguez $^{\mathrm{b}, 4}$, \\ Pilar Salgado a,1 \\ ${ }^{a}$ Departamento de Matemática Aplicada, Universidade de Santiago de Compostela, 15782 Santiago de Compostela, Spain \\ ${ }^{\mathrm{b}} \mathrm{CI}^{2} \mathrm{MA}$, Departamento de Ingeniería Matemática, Universidad de Concepción, Chile
}

\begin{abstract}
The goal of this Note is to prove that two, in principle different, well-known finite element approximations of the eddy current model are equivalent. The first one concerns a formulation involving the magnetic field in the conductor and the magnetic scalar potential in the dielectric. The second one solves another formulation of the same problem involving the magnetic field in both, the conductor and the dielectric, and a Lagrange multiplier in the dielectric. The latter is also shown to be equivalent to a third formulation involving two Lagrange multipliers, which leads to a well posed linear system.
\end{abstract}

\section{Résumé}

Équivalence entre deux méthodes d'éléments finis pour le problème des courants de Foucault. Le but de cette Note est de démontrer que deux méthodes d'éléments finis pour la résolution du problème des courants de Foucault, en principe différentes, sont complètement équivalentes. La premiére concerne une formulation qui a comme inconnues principales le champ magnétique dans le conducteur et le potentiel scalaire magnétique dans le diélectrique. La seconde résout une formulation qui inclut le champ magnétique dans tout le domaine et un multiplicateur de Lagrange dans le diélectrique. On démontre aussi que celle-ci est équivalente à une trosième formulation avec deux multiplicateurs de Lagrange, qui conduit à un système linéaire bien posé.

\footnotetext{
‡ Work partially supported by Ministerio de Ciencia e Innovación of Spain under grant number MTM2008-02483.

Email addresses: alfredo.bermudez@usc.es (Alfredo Bermúdez), blopezr@unal.edu.co (Bibiana López-Rodríguez), rodolfo@ing-mat.udec.cl (Rodolfo Rodríguez), mpilar.salgado@usc.es (Pilar Salgado).

1 Partially supported by Xunta de Galicia under grant number INCITE09-207047-PR.

2 Partially supported by a MECESUP fellowship (Chile) and a Banco Santander-USC fellowship (Spain).

3 Permanent address : Universidad Nacional de Colombia, Sede Medellín, Colombia.

4 Partially supported by FONDAP and BASAL projects, CMM, (Chile).
}

Preprint submitted to Elsevier Science 


\section{Version française abrégée}

Cette Note concerne la résolution par éléments finis du problème des courants de Foucault (1) avec les conditions aux limites (2). Ce problème a été considéré dans [4] où une formulation en champ magnétique dans le conducteur et en potentiel scalaire magnétique dans le diélectrique a été analysée. Pour la résolution numérique de cette formulation on y a proposé des éléments de Nédélec pour l'approximation du champ magnétique et des fonctions continues, linéaires par élément fini, pour le potentiel scalaire magnétique (voir le Problème 3). Des estimations d'erreur optimales ont été obtenues et confirmées para les experiences numériques.

Cette méthodologie pour la résolution du problème des courants de Foucault est intéressante du point de vue du temps de calcul, dans la mesure où on a seulement une inconnue scalaire dans le diélectrique. Cependant, très souvant le diélectrique n'est pas simplemente connexe et l'implementation sur ordinateur a besoin de coupures dans le maillage (voir [2], [4]), ce qui est une difficulté d'ordre pratique.

Une possibilité pour l'éviter consiste à utiliser un multiplicateur de Lagrange pour imposer le caractère solenoidal du champ magnétique dans le domaine diélectrique. Cette alternative a été analysée dans [1] aux niveaux continu et discret, dans un cas avec des conditions aux limites différentes des (2).

Dans cette Note nous introduisons cette méthode au niveau discret. Plus précisément, nous considérons le Problème 5 où le champ magnétique est approché par des éléments finis de Nédélec, à la fois dans le conducteur et dans le diélectrique, tandis que le multiplicateur de Lagrange est pris dans l'espace des rotationnels des éléments de Nédélec. Le but principal de cette Note est de montrer que ce problème discret est complètement équivalent au Problème 3, dans ce sens que les champs magnétiques solutions des deux problèmes sont exactement les mêmes. Plus précisément on a le résultat suivant :

Proposition 0.1 Étant donné $\boldsymbol{I} \in \mathbb{C}^{N}$, un champ discret $\boldsymbol{H}_{h} \in \mathcal{N}_{h}(\Omega)$ est solution du Problème 2 (de façon équivalente, du Problème 3) si, et seulement si, il existe $\boldsymbol{Z}_{h} \in \operatorname{curl}\left(\mathcal{N}_{h}\left(\Omega_{\mathrm{D}}\right)\right)$ tel que $\left(\boldsymbol{H}_{h}, \boldsymbol{Z}_{h}\right)$ est solution du Problème 5.

La preuve de ce resultat est basée sur la vérification d'une condition inf-sup discrète qui est donnée dans la Proposition 3.1.

Finalement, puisque l'implementation de la seconde formulation conduit à un système à matrice singulière, nous démontrons aussi l'équivalence avec une troisème formulation qui nous amène à un système d'équations linéaires bien posé.

\section{Introduction}

We start introducing the time-harmonic eddy current problem:

$$
\operatorname{curl} \boldsymbol{H}=\boldsymbol{J}, \quad \mathrm{i} \omega \mu \boldsymbol{H}+\operatorname{curl} \boldsymbol{E}=\mathbf{0}, \quad \operatorname{div}(\mu \boldsymbol{H})=0, \quad \boldsymbol{J}=\sigma \boldsymbol{E},
$$

where $\boldsymbol{E}$ is the electric field, $\boldsymbol{H}$ the magnetic field, $\boldsymbol{J}$ the current density, $\omega$ the angular frequency, $\mu$ the magnetic permeability and $\sigma$ the electric conductivity. We are interested in solving these equations in a simply connected three-dimensional bounded domain $\Omega$, which consists of two parts, $\Omega_{\mathrm{C}}$ and $\Omega_{\mathrm{D}}$, occupied by conductors and dielectrics, respectively. The electric conductivity $\sigma$ vanishes in the dielectric domain. We denote $\Omega_{\mathrm{C}}^{1}, \ldots, \Omega_{\mathrm{C}}^{N}$ the connected components of $\Omega_{\mathrm{C}}$. The domain $\Omega$ is assumed to have a Lipschitzcontinuous connected boundary $\partial \Omega$, which splits into two parts: $\partial \Omega=\Gamma_{\mathrm{C}} \cup \Gamma_{\mathrm{D}}$, with $\Gamma_{\mathrm{C}}:=\partial \Omega_{\mathrm{C}} \cap \partial \Omega$ and $\Gamma_{\mathrm{D}}:=\partial \Omega_{\mathrm{D}} \cap \partial \Omega$ being the outer boundaries of the conducting and dielectric domains, respectively. We denote $\Gamma_{\mathrm{I}}:=\partial \Omega_{\mathrm{C}} \cap \partial \Omega_{\mathrm{D}}$, the interface between dielectrics and conductors. We also denote by $\boldsymbol{n}$ and $\boldsymbol{n}_{\mathrm{C}}$ the outer unit normal vectors to $\partial \Omega$ and $\partial \Omega_{\mathrm{C}}$, respectively. We assume that the outer boundary of each connected component, $\partial \Omega_{\mathrm{C}}^{n} \cap \partial \Omega(n=1, \ldots, N)$, has two connected components, both with non-zero 
measure: the current entrance, $\Gamma_{J}^{n}$, where it is connected to a wire supplying alternating electric current, and the current free exit, $\Gamma_{E}^{n}$. Finally, we denote $\Gamma_{J}:=\Gamma_{J}^{1} \cup \cdots \cup \Gamma_{J}^{N}$ and $\Gamma_{E}:=\Gamma_{E}^{1} \cup \cdots \cup \Gamma_{E}^{N}$, and we assume that $\Gamma_{J} \cap \Gamma_{E}=\emptyset$. (See a sketch of the domain in Figure 1.)
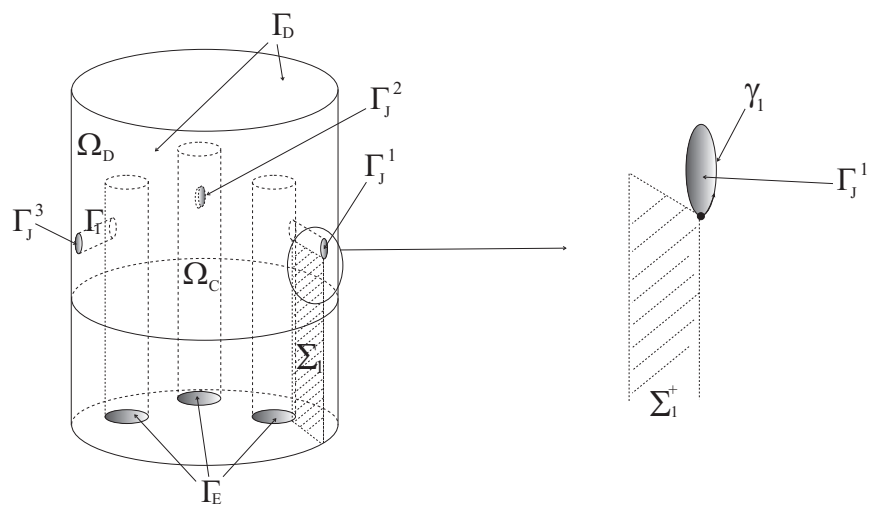

Figure 1. Sketch of the domain (left) and zoom around the current entrance (right).

According to [5], the following boundary conditions have been considered in [4]:

$$
\boldsymbol{E} \times \boldsymbol{n}=\mathbf{0} \quad \text { on } \Gamma_{\boldsymbol{E}} \cup \Gamma_{J}, \quad \int_{\Gamma_{J}^{n}} \operatorname{curl} \boldsymbol{H} \cdot \boldsymbol{n}=I_{n} \quad \text { on } \Gamma_{J}^{n}, n=1, \ldots, N, \quad \mu \boldsymbol{H} \cdot \boldsymbol{n}=0 \quad \text { on } \partial \Omega,
$$

where the only data are the current intensities $I_{n}$ through each wire.

In this Note we consider two different formulations of the above problem. The first one uses the magnetic field in the conductor and a magnetic scalar potential in the dielectric. The second one involves the magnetic field in the whole domain, and a vector Lagrange multiplier in the dielectric. We prove that two well-known finite element discretizations, one of each formulation, are actually equivalent. Moreover, since the direct implementation of the second formulation leads to a linear system with a singular matrix, we also show the equivalence with a third one which leads to a well-posed system of linear equations.

\section{The magnetic field/magnetic scalar potential formulation}

Let $\mathcal{X}:=\left\{\boldsymbol{G} \in \mathrm{H}(\operatorname{curl}, \Omega): \operatorname{curl} \boldsymbol{G}=\mathbf{0}\right.$ in $\left.\Omega_{\mathrm{D}}\right\}$ and $a: \mathrm{H}(\operatorname{curl}, \Omega) \times \mathrm{H}(\operatorname{curl}, \Omega) \longrightarrow \mathbb{C}$ be the sesquilinear continuous form defined by $a(\boldsymbol{H}, \boldsymbol{G}):=\mathrm{i} \omega \int_{\Omega} \mu \boldsymbol{H} \cdot \overline{\boldsymbol{G}}+\int_{\Omega_{\mathrm{C}}} \frac{1}{\sigma} \operatorname{curl} \boldsymbol{H} \cdot \operatorname{curl} \overline{\boldsymbol{G}}$. Given $\boldsymbol{I}:=\left(I_{n}\right) \in \mathbb{C}^{N}$, we introduce the closed linear manifold of $\mathcal{X}$ defined as follows: $\mathcal{V}(\boldsymbol{I}):=\left\{\boldsymbol{G} \in \mathcal{X}:\langle\operatorname{curl} \boldsymbol{G} \cdot \boldsymbol{n}, 1\rangle_{\Gamma_{J}^{n}}=\right.$ $\left.I_{n}, n=1, \ldots, N\right\}$.

The following weak formulation of the above eddy current problem has been obtained in [4]:

Problem 1 Given $\boldsymbol{I} \in \mathbb{C}^{N}$, find $\boldsymbol{H} \in \mathcal{V}(\boldsymbol{I})$ such that a $(\boldsymbol{H}, \boldsymbol{G})=0 \forall \boldsymbol{G} \in \mathcal{V}(\mathbf{0})$.

For the numerical solution let us assume that $\Omega, \Omega_{\mathrm{C}}$ and $\Omega_{\mathrm{D}}$ are Lipschitz polyhedra and consider regular tetrahedral meshes $\mathcal{T}_{h}$ of $\Omega$, such that each element $K \in \mathcal{T}_{h}$ is contained either in $\Omega_{\mathrm{C}}$ or in $\Omega_{\mathrm{D}}$ ( $h$ stands as usual for the corresponding mesh-size). We employ "edge" finite elements to approximate the magnetic field. More precisely, the lowest-order Nédélec finite element space $\mathcal{N}_{h}(\Omega)$ (see, for instance [7]). We introduce the finite-dimensional space $\mathcal{X}_{h}:=\left\{\boldsymbol{G}_{h} \in \mathcal{N}_{h}(\Omega): \operatorname{curl} \boldsymbol{G}_{h}=\mathbf{0}\right.$ in $\left.\Omega_{\mathrm{D}}\right\} \subset \mathcal{X}$ and the linear manifolds $\mathcal{V}_{h}(\boldsymbol{I}):=\mathcal{V}(\boldsymbol{I}) \cap \mathcal{X}_{h}, \boldsymbol{I} \in \mathbb{C}^{N}$. Then, the discrete problem reads as follows. 
Problem 2 Given $\boldsymbol{I} \in \mathbb{C}^{N}$, find $\boldsymbol{H}_{h} \in \mathcal{V}_{h}(\boldsymbol{I})$ such that $a\left(\boldsymbol{H}_{h}, \boldsymbol{G}_{h}\right)=0 \forall \boldsymbol{G}_{h} \in \mathcal{V}_{h}(\mathbf{0})$.

In [4] it is proved that this problem has a unique solution which is an optimal order approximation of the solution to Problem 1. In what follows we recall how to impose efficiently the curl-free condition in the definition of $\boldsymbol{\mathcal { X }}_{h}$ by introducing a discrete multivalued magnetic potential in the dielectric domain.

Assume that for each connected component of the conducting domain, $\Omega_{\mathrm{C}}^{n}$, there exists a connected "cut" surface $\Sigma_{n} \subset \Omega_{\mathrm{D}}$ such that $\partial \Sigma_{n} \subset \partial \Omega_{\mathrm{D}}$ and $\widetilde{\Omega}_{\mathrm{D}}:=\Omega_{\mathrm{D}} \backslash \bigcup_{n=1}^{N} \Sigma_{n}$ is pseudo-Lipschitz and simply connected (see, for instance, [2]). Also assume that $\Sigma_{n} \cap \bar{\Sigma}_{m}=\emptyset$ for $n \neq m$ and that the boundary of each current entrance surface, $\Gamma_{J}^{n}$, is a simple closed curve, that we denote $\gamma_{n}$.

We denote the two faces of each $\Sigma_{n}$ by $\Sigma_{n}^{-}$and $\Sigma_{n}^{+}$, and fix a unit normal $\boldsymbol{n}_{n}$ on $\Sigma_{n}$ as the "outer" normal to $\Omega_{\mathrm{D}} \backslash \Sigma_{n}$ along $\Sigma_{n}^{+}$. We choose an orientation for each $\gamma_{n}$ by taking its initial and end points on $\Sigma_{n}^{-}$and $\Sigma_{n}^{+}$, respectively. We denote by $\boldsymbol{t}_{n}$ the unit vector tangent to $\gamma_{n}$. (See Figure 1.)

For any function $\widetilde{\Psi} \in \mathrm{H}^{1}\left(\widetilde{\Omega}_{\mathrm{D}}\right)$, we denote by $\llbracket \widetilde{\Psi} \rrbracket_{\Sigma_{n}}:=\left.\widetilde{\Psi}\right|_{\Sigma_{n}^{-}}-\left.\widetilde{\Psi}\right|_{\Sigma_{n}^{+}}$the jump of $\widetilde{\Psi}$ through $\Sigma_{n}$ along $\boldsymbol{n}_{n}$. The gradient of $\widetilde{\Psi}$ in $\mathcal{D}^{\prime}\left(\widetilde{\Omega}_{\mathrm{D}}\right)$ can be extended to $\mathrm{L}^{2}\left(\Omega_{\mathrm{D}}\right)^{3}$ and will be denoted by grad $\widetilde{\Psi}$.

Assume that the cut surfaces $\Sigma_{n}$ are polyhedral and the meshes are compatible with them, in the sense that each $\Sigma_{n}$ is a union of faces of tetrahedra $K \in \mathcal{T}_{h}$. Therefore, $\mathcal{T}_{h}^{\Omega_{\mathrm{D}}}:=\left\{K \in \mathcal{T}_{h}: K \subset \Omega_{\mathrm{D}}\right\}$ can also be seen as a mesh of $\widetilde{\Omega}_{\mathrm{D}}$.

Let us introduce the following discrete spaces: $\mathcal{L}_{h}\left(\widetilde{\Omega}_{\mathrm{D}}\right):=\left\{\widetilde{\Psi}_{h} \in \mathrm{H}^{1}\left(\widetilde{\Omega}_{\mathrm{D}}\right):\left.\widetilde{\Psi}_{h}\right|_{K} \in \mathcal{P}_{1}(K) \forall K \in \mathcal{T}_{h}^{\Omega_{\mathrm{D}}}\right\}$, $\Theta_{h}:=\left\{\widetilde{\Psi}_{h} \in \mathcal{L}_{h}\left(\widetilde{\Omega}_{\mathrm{D}}\right): \llbracket \widetilde{\Psi}_{h} \rrbracket_{\Sigma_{n}}=\right.$ constant, $\left.n=1, \ldots N\right\}$ and $\mathcal{Y}_{h}:=\left\{\left(\boldsymbol{G}_{h}, \widetilde{\Psi}_{h}\right) \in \mathcal{N}_{h}\left(\Omega_{\mathrm{C}}\right) \times\left(\Theta_{h} / \mathbb{C}\right):\right.$ $\boldsymbol{G}_{h} \times \boldsymbol{n}_{\mathrm{C}}=\operatorname{grad} \widetilde{\Psi}_{h} \times \boldsymbol{n}_{\mathrm{C}}$ on $\left.\Gamma_{\mathrm{I}}\right\}$. Let $\mathcal{W}_{h}(\boldsymbol{I}):=\left\{\left(\boldsymbol{G}_{h}, \widetilde{\Psi}_{h}\right) \in \mathcal{Y}_{h}: \llbracket \widetilde{\Psi}_{h} \rrbracket_{\Sigma_{n}}=I_{n}, n=1, \ldots, N\right\}, \boldsymbol{I} \in \mathbb{C}^{N}$, and $\widetilde{a}\left(\left(\boldsymbol{H}_{h}, \widetilde{\Phi}_{h}\right),\left(\boldsymbol{G}_{h}, \widetilde{\Psi}_{h}\right)\right):=\mathrm{i} \omega \int_{\Omega_{\mathrm{D}}} \mu \operatorname{grad} \widetilde{\Phi}_{h} \cdot \operatorname{grad} \widetilde{\widetilde{\Psi}}_{h}+\mathrm{i} \omega \int_{\Omega_{\mathrm{C}}} \mu \boldsymbol{H}_{h} \cdot \overline{\boldsymbol{G}}_{h}+\int_{\Omega_{\mathrm{C}}} \frac{1}{\sigma} \operatorname{curl} \boldsymbol{H}_{h} \cdot \operatorname{curl} \overline{\boldsymbol{G}}_{h}$, $\left(\boldsymbol{H}_{h}, \widetilde{\Phi}_{h}\right),\left(\boldsymbol{G}_{h}, \widetilde{\Psi}_{h}\right) \in \mathcal{Y}_{h}$. Now, consider the following discrete problem.

Problem 3 Given $\boldsymbol{I} \in \mathbb{C}^{N}$, find $\left(\boldsymbol{H}_{h}, \widetilde{\Phi}_{h}\right) \in \mathcal{W}_{h}(\boldsymbol{I})$ such that $\widetilde{a}\left(\left(\boldsymbol{H}_{h}, \widetilde{\Phi}_{h}\right),\left(\boldsymbol{G}_{h}, \widetilde{\Psi}_{h}\right)\right)=0 \forall\left(\boldsymbol{G}_{h}, \widetilde{\Psi}_{h}\right) \in$ $\mathcal{W}_{h}(\mathbf{0})$.

The next theorem, proved in [4], shows that Problems 2 and 3 are equivalent.

Theorem 4 Given $\boldsymbol{I} \in \mathbb{C}^{N}, \boldsymbol{H}_{\widetilde{T}}$ is a solution of Problem 2 if and only if there exists $\widetilde{\Phi}_{h} \in \Theta_{h}$ such that $\left.\boldsymbol{H}_{h}\right|_{\Omega_{\mathrm{D}}}=\underset{\operatorname{grad}}{ } \widetilde{\Phi}_{h}$ and $\left(\left.\boldsymbol{H}_{h}\right|_{\Omega_{\mathrm{C}}}, \widetilde{\Phi}_{h}\right)$ is a solution of Problem 3.

Problem 3 leads to an important saving in computational effort, since it involves a scalar instead of a vector field in the dielectric. However, its implementation requires imposing the following constraints:

- $\boldsymbol{G}_{h} \times \boldsymbol{n}_{\mathrm{C}}=\boldsymbol{\operatorname { g r a d }} \widetilde{\Psi}_{h} \times \boldsymbol{n}_{\mathrm{C}}$ on $\Gamma_{\mathrm{I}}$, which arises in the definition of $\mathcal{Y}_{h}$;

- $\llbracket \widetilde{\Psi}_{h} \rrbracket_{\Sigma_{n}}=$ constant, $n=1, \ldots, N$, which arise in the definition of $\Theta_{h}$.

A procedure to impose these constraints was proposed in [3], where numerical experiments which exhibit the performance of the method were also reported.

\section{A discrete mixed formulation}

Solving Problem 3 is a good alternative to obtain an approximate solution of the eddy current model. The only drawback is that it needs finite element meshes involving cuts, which sometimes can be difficult to build. In what follows we will introduce a mixed discrete formulation of the same eddy current model given above, which does not need any cut, and we will show that it is completely equivalent to Problem 2.

This mixed formulation has been previously analyzed in [1] for other boundary conditions, without establishing any relation with a magnetic field/magnetic scalar potential discretization as that of Problem 2 and, consequently, without taking advantage of the equivalence between such discrete problems. 
The formulation is based on using a Lagrange multiplier to impose the curl-free constraint in the dielectric instead of introducing the scalar potential in $\Omega_{\mathrm{D}}$, so that cuts are not required in the mesh.

Let $\mathcal{U}_{h}(\boldsymbol{I}):=\left\{\boldsymbol{G}_{h} \in \mathcal{N}_{h}(\Omega): \int_{\Gamma_{\boldsymbol{J}}^{n}} \operatorname{curl} \boldsymbol{G}_{h} \cdot \boldsymbol{n}=I_{n}, n=1, \ldots, N\right\}, \boldsymbol{I} \in \mathbb{C}^{N}$. The discrete mixed problem reads as follows.

Problem 5 Given $\boldsymbol{I} \in \mathbb{C}^{N}$, find $\boldsymbol{H}_{h} \in \mathcal{U}_{h}(\boldsymbol{I})$ and $\boldsymbol{Z}_{h} \in \operatorname{curl}\left(\mathcal{N}_{h}\left(\Omega_{\mathrm{D}}\right)\right)$ such that

$$
\begin{array}{lr}
a\left(\boldsymbol{H}_{h}, \boldsymbol{G}_{h}\right)+\int_{\Omega_{\mathrm{D}}} \boldsymbol{Z}_{h} \cdot \operatorname{curl} \overline{\boldsymbol{G}}_{h}=0 & \forall \boldsymbol{G}_{h} \in \mathcal{U}_{h}(\mathbf{0}), \\
\int_{\Omega_{\mathrm{D}}} \operatorname{curl} \boldsymbol{H}_{h} \cdot \overline{\boldsymbol{F}}_{h}=0 & \forall \boldsymbol{F}_{h} \in \operatorname{curl}\left(\mathcal{N}_{h}\left(\Omega_{\mathrm{D}}\right)\right) .
\end{array}
$$

Since $\left\{\boldsymbol{G}_{h} \in \mathcal{U}_{h}(\mathbf{0}): \int_{\Omega_{\mathrm{D}}} \operatorname{curl} \boldsymbol{G}_{h} \cdot \overline{\boldsymbol{F}}_{h}=0 \forall \boldsymbol{F}_{h} \in \operatorname{curl}\left(\mathcal{N}_{h}\left(\Omega_{\mathrm{D}}\right)\right)\right\}=\mathcal{U}_{h}(\mathbf{0}) \cap \mathcal{X}$ and $a$ is coercive on $\mathcal{X}$, we only need the following inf-sup condition to conclude that Problem 5 has a unique solution.

Proposition 3.1 There exist a constant $\beta_{h}>0$ such that

$$
\sup _{\boldsymbol{G}_{h} \in \mathcal{U}_{h}(\mathbf{0}): \boldsymbol{G}_{h} \neq \mathbf{0}} \frac{\left|\int_{\Omega_{\mathrm{D}}} \boldsymbol{F}_{h} \cdot \operatorname{curl} \overline{\boldsymbol{G}}_{h}\right|}{\left\|\boldsymbol{G}_{h}\right\|_{\mathrm{H}(\operatorname{curl}, \Omega)}} \geq \beta_{h}\left\|\boldsymbol{F}_{h}\right\|_{\mathrm{L}^{2}\left(\Omega_{\mathrm{D}}\right)} \quad \forall \boldsymbol{F}_{h} \in \operatorname{curl}\left(\mathcal{N}_{h}\left(\Omega_{\mathrm{D}}\right)\right) .
$$

Proof. Since $\operatorname{dim} \mathcal{U}_{h}(\mathbf{0})<\infty$, it is enough to prove that for all non-vanishing $\boldsymbol{F}_{h} \in \operatorname{curl}\left(\mathcal{N}_{h}\left(\Omega_{\mathrm{D}}\right)\right)$ there exists $\boldsymbol{G}_{h} \in \boldsymbol{U}_{h}(\mathbf{0})$ such that $\int_{\Omega_{\mathrm{D}}} \boldsymbol{F}_{h} \cdot \operatorname{curl} \boldsymbol{G}_{h} \neq 0$. Let $\boldsymbol{U}_{h} \in \mathcal{N}_{h}(\Omega)$ be such that $\boldsymbol{F}_{h}=\operatorname{curl} \boldsymbol{U}_{h}$ in $\Omega_{\mathrm{D}}$. In general $\boldsymbol{U}_{h} \notin \mathcal{U}_{h}(\mathbf{0})$, but if we are able to find $\boldsymbol{Y}_{h} \in \mathcal{N}_{h}(\Omega)$ satisfying $\operatorname{curl} \boldsymbol{Y}_{h}=\mathbf{0}$ in $\Omega_{\mathrm{D}}$ and $\int_{\gamma_{n}} \boldsymbol{Y}_{h} \cdot \boldsymbol{t}_{n}=$ $-\int_{\gamma_{n}} \boldsymbol{U}_{h} \cdot \boldsymbol{t}_{n}, n=1, \ldots, N$, it is straightforward to check that $\boldsymbol{G}_{h}:=\boldsymbol{U}_{h}+\boldsymbol{Y}_{h}$ satisfies the above requirements (recall $\left.\gamma_{n}:=\partial \Gamma_{J}^{n}\right)$. Such a $\boldsymbol{Y}_{h}$ can be defined as follows: $\boldsymbol{Y}_{h}:=-\sum_{m=1}^{N}\left(\int_{\gamma_{m}} \boldsymbol{U}_{h} \cdot \boldsymbol{t}_{m}\right) \boldsymbol{Y}_{h}^{m}$, where $\boldsymbol{Y}_{h}^{m} \in \mathcal{N}_{h}(\Omega)$ is such that $\left.\boldsymbol{Y}_{h}^{m}\right|_{\Omega_{\mathrm{D}}}=\operatorname{grad} \widetilde{\Phi}_{h}^{m}$, with $\widetilde{\Phi}_{h}^{m} \in \Theta_{h}$ satisfying $\llbracket \widetilde{\Phi}_{h}^{m} \rrbracket_{\Sigma_{n}}=\delta_{n m}, n, m=$ $1, \ldots, N$.

An inf-sup condition analogous to that in Proposition 3.1 has been proved to hold uniformly in $h$ in the proof of Theorem 5.2 from [1] for the problem considered in that paper. However, this is not necessary, at least in our case, since we will obtain error estimates for the component $\boldsymbol{H}_{h}$ of the solution to Problem 5 as a direct consequence of the following equivalence result.

Proposition 3.2 Given $\boldsymbol{I} \in \mathbb{C}^{N}$, a discrete field $\boldsymbol{H}_{h} \in \mathcal{N}_{h}(\Omega)$ is solution of Problem 2 (equivalently, of Problem 3) if and only if there exists $\boldsymbol{Z}_{h} \in \operatorname{curl}\left(\mathcal{N}_{h}\left(\Omega_{\mathrm{D}}\right)\right)$ such that $\left(\boldsymbol{H}_{h}, \boldsymbol{Z}_{h}\right)$ solves Problem 5.

Proof. Since each problem has a unique solution, it is enough to prove that if $\left(\boldsymbol{H}_{h}, \boldsymbol{Z}_{h}\right)$ solves Problem 5 , then $\boldsymbol{H}_{h}$ solves Problem 2. For this purpose, let us take $\boldsymbol{F}_{h}=\operatorname{curl} \boldsymbol{H}_{h}$ as test function in (4). We deduce $\operatorname{curl} \boldsymbol{H}_{h}=\mathbf{0}$ and then $\boldsymbol{H}_{h} \in \mathcal{V}_{h}(\boldsymbol{I})$. Finally, we complete the proof by testing (3) with $\boldsymbol{G}_{h} \in \mathcal{V}_{h}(\mathbf{0})$.

Although Problem 5 has a unique solution, its direct implementation leads to a singular linear system. Indeed, when the functions $\boldsymbol{F}_{h} \in \operatorname{curl}\left(\mathcal{N}_{h}\left(\Omega_{\mathrm{D}}\right)\right)$ are written as $\boldsymbol{F}_{h}=\operatorname{curl} \boldsymbol{U}_{h}$, with $\boldsymbol{U}_{h} \in \mathcal{N}_{h}\left(\Omega_{\mathrm{D}}\right)$, such $\boldsymbol{U}_{h}$ is clearly not unique and this leads to a singular matrix. However, as stated in [1, Remark 5.1], since the kernel of this matrix is well separated from the rest of the spectrum, a conjugate gradient type method will work for its numerical solution.

An alternative leading to a system with a non-singular matrix, was also proposed in [1]. Let $\mathcal{Q}_{h}$ be the space of piecewise constant functions in $\mathcal{T}_{h}^{\Omega_{\mathrm{D}}}$ and $\mathcal{C R}_{h}^{0}\left(\Omega_{\mathrm{D}}\right)$ the space of lowest-order 3D CrouzeixRaviart elements (see [1], for instance) that vanish at the mid-points of faces lying on $\partial \Omega_{\mathrm{D}}$ (for simplicity, we assume $\partial \Omega_{\mathrm{D}}$ connected). 
The functions in $\mathcal{C R}_{h}^{0}\left(\Omega_{\mathrm{D}}\right)$ are piecewise linear in $\mathcal{T}_{h}^{\Omega_{\mathrm{D}}}$ and continuous at the mid-points of each face. For $q_{h} \in \mathcal{C R}_{h}^{0}\left(\Omega_{\mathrm{D}}\right)$, let $\widehat{\operatorname{grad}} q_{h}$ denote the vector field in $\mathcal{Q}_{h}^{3}$ defined by $\left.\left(\widehat{\operatorname{grad}} q_{h}\right)\right|_{K}:=\operatorname{grad}\left(\left.q_{h}\right|_{K}\right)$, $K \in \mathcal{T}_{h}^{\Omega_{\mathrm{D}}}$. The following result has been proved in [6, Theorem 4.9] (see also [1, Lemma 5.4] for $\partial \Omega_{\mathrm{D}}$ non connected).

Lemma 3.1 $\mathcal{Q}_{h}^{3}=\operatorname{curl}\left(\mathcal{N}_{h}\left(\Omega_{\mathrm{D}}\right)\right) \oplus \widehat{\operatorname{grad}}\left(\mathcal{C R}_{h}^{0}\left(\Omega_{\mathrm{D}}\right)\right)$ and the decomposition is orthogonal in $L^{2}\left(\Omega_{\mathrm{D}}\right)^{3}$.

Consider the following discrete problem.

Problem 6 Given $\boldsymbol{I} \in \mathbb{C}^{N}$, find $\boldsymbol{H}_{h} \in \mathcal{U}_{h}(\boldsymbol{I}), \boldsymbol{Z}_{h} \in \mathcal{Q}_{h}^{3}$ and $p_{h} \in \mathcal{C R}_{h}^{0}\left(\Omega_{\mathrm{D}}\right)$ such that

$$
\begin{array}{lr}
a\left(\boldsymbol{H}_{h}, \boldsymbol{G}_{h}\right)+\int_{\Omega_{\mathrm{D}}} \boldsymbol{Z}_{h} \cdot \operatorname{curl} \overline{\boldsymbol{G}}_{h}=0 & \forall \boldsymbol{G}_{h} \in \mathcal{U}_{h}(\mathbf{0}), \\
\int_{\Omega_{\mathrm{D}}} \operatorname{curl} \boldsymbol{H}_{h} \cdot \overline{\boldsymbol{F}}_{h}+\int_{\Omega_{\mathrm{D}}} \widehat{\operatorname{grad}} p_{h} \cdot \overline{\boldsymbol{F}}_{h}=0 & \forall \boldsymbol{F}_{h} \in \mathcal{Q}_{h}^{3}, \\
\int_{\Omega_{\mathrm{D}}} \boldsymbol{Z}_{h} \cdot \widehat{\operatorname{grad}} \bar{q}_{h}=0 & \forall q_{h} \in \mathcal{C \mathcal { R }}_{h}^{0}\left(\Omega_{\mathrm{D}}\right) .
\end{array}
$$

Next result shows that Problems 6 is equivalent to Problems 5 and, hence, to Problems 2 and 3 too.

Proposition 3.3 Let $\boldsymbol{I} \in \mathbb{C}^{N}$. If $\left(\boldsymbol{H}_{h}, \boldsymbol{Z}_{h}\right)$ is solution of Problem 5, then $\left(\boldsymbol{H}_{h}, \boldsymbol{Z}_{h}, 0\right)$ solves Problem 6. Conversely, if $\left(\boldsymbol{H}_{h}, \boldsymbol{Z}_{h}, p_{h}\right)$ solves Problem 6, then $p_{h}=0$ and $\left(\boldsymbol{H}_{h}, \boldsymbol{Z}_{h}\right)$ is solution of Problem 5.

Proof. Let $\left(\boldsymbol{H}_{h}, \boldsymbol{Z}_{h}\right)$ be solution of Problem 5. Then $\left(\boldsymbol{H}_{h}, \boldsymbol{Z}_{h}, 0\right)$ satisfies (5) and (6), the latter by virtue of Lemma 3.1. On the other hand, (7) follows from the fact that $\boldsymbol{Z}_{h} \in \operatorname{curl}\left(\mathcal{N}_{h}\left(\Omega_{\mathrm{D}}\right)\right)$ and Lemma 3.1 again. Conversely, let $\left(\boldsymbol{H}_{h}, \boldsymbol{Z}_{h}, p_{h}\right)$ be solution of Problem 6. By testing (6) with $\boldsymbol{F}_{h}=\widehat{\operatorname{grad}} p_{h}$, it follows from Lemma 3.1 that $p_{h}=0$. The same lemma and (7) imply that $\boldsymbol{Z}_{h} \in \operatorname{curl}\left(\mathcal{N}_{h}\left(\Omega_{\mathrm{D}}\right)\right)$. Hence, for $p_{h}=0,(5)$ and (6) shows that $\left(\boldsymbol{H}_{h}, \boldsymbol{Z}_{h}\right)$ solves Problem 5 .

As a consequence of the above proposition and the well-posedness of Problem 5, it follows that Problem 6 has also a unique solution. Thus, using standard basis for the finite element spaces leads to a linear system with a non-singular matrix. On the other hand, the approximation properties proved in [4] for Problem 2, automatically lead to optimal order error estimates for the component $\boldsymbol{H}_{h}$ of the solution to Problem 6 .

We have implemented the numerical methods described above and the numerical tests confirm all the theoretical results.

\section{References}

[1] A. Alonso Rodriguez, R. Hiptmair, A. Valli, Mixed finite element approximation of eddy current problems, IMA J. Numer. Anal. 24 (2004) 255-271.

[2] C. Amrouche, C. Bernardi, M. Dauge, V. Girault, Vector potentials in three-dimensional non-smooth domains, Math. Methods Appl. Sci. 21 (1998) 823-864.

[3] A. Bermúdez, R. Rodríguez, P. Salgado, A finite element method with Lagrange multipliers for low-frequency harmonic Maxwell equations, SIAM J. Numer. Anal. 40 (2002) 1823-1849.

[4] A. Bermúdez, R. Rodríguez, P. Salgado, Numerical solution of eddy current problems in bounded domains using realistic boundary conditions. Comput. Methods Appl. Mech. Engrg. 194 (2005) 411-426.

[5] A. Bossavit, Most general "non-local" boundary conditions for the Maxwell equation in a bounded region, COMPEL 19 (2000) 3239-3245.

[6] P. Monk, A mixed method for approximating Maxwell's equations, SIAM J. Numer. Anal. 28 (1991) 1610-1634.

[7] P. Monk, Finite element methods for Maxwell's equations, Oxford University Press, New York, 2003. 